\title{
Approximate treatment of a two-dimensional anisotropic Peierls-Hubbard model
}

\author{
B. H. Bernhard \\ Laboratoire de Physique des Solides, Batiment 510, Universite de Paris-Sud, 91405 Orsay CEDEX, France \\ and Instituto de Física, Universidade Federal do Rio Grande do Sul, Caixa Postal 15051, 91501-970 Porto Alegre, \\ Rio Grande do Sul, Brazil \\ J. R. Iglesias \\ Instituto de Física, Universidade Federal do Rio Grande do Sul, Caixa Postal 15051, 91501-970 Porto Alegre, \\ Rio Grande do Sul, Brazil
}

(Received 8 June 1992; revised manuscript received 24 November 1992)

\begin{abstract}
A Green-function formalism is presented to study a Peierls-Hubbard Hamiltonian in two dimensions. The lattice consists of parallel dimerized chains with alternating nearest-neighbor hoppings $t$ and $t_{\|}$and another hopping $t_{\perp}$ between different chains. The method treats the interdimer hopping as a perturbation and yields exact results in the uncorrelated case and for isolated dimers. The calculated spectral functions exhibit a number of narrow subbands with typical low-dimensional singularities. The dependence of the gap at the Fermi level on the electron-electron interaction $U$ agrees qualitatively with the exact result in the known one-dimensional nondimerized limit. The paramagnetic susceptibility shows a maximum structure at low temperatures that is enhanced by $U$ and by dimerization and a Curie-Weiss behavior at high temperatures.
\end{abstract}

\section{INTRODUCTION}

In spite of the cumulative effort directed to the study of the Hubbard Hamiltonian ${ }^{1}$ and its apparent simplicity, few exact results have been obtained so far, namely, for the linear chain ${ }^{2}$ and small clusters. ${ }^{3-5}$ A renewed interest in it in connection to high- $T_{c}$ superconductivity ${ }^{6}$ inspired the reexamination of standard treatments to higher order. An example is the many-body perturbation theory with respect to Coulomb repulsion $U$ that showed some new features such as satellite peaks in the spectral functions. ${ }^{7}$ Different extensions of the Hamiltonian have been considered, taking into account the effect of longrange Coulomb repulsion, next-nearest-neighbor hopping, and multiple bands. ${ }^{8}$ In the strong-coupling limit it gave rise to the $t-J$ model, ${ }^{9}$ where doubly occupied states are projected out.

Important information about properties of the Hubbard model and $t-J$ model can be obtained by exactdiagonalization techniques ${ }^{10,11}$ and Monte Carlo simulations $^{12-15}$ on finite clusters of increasing size. Nevertheless, in the low-temperature region finite-size effects become important and much larger systems are required to extrapolate the results to the thermodynamic limit.

The introduction of electron-phonon interaction into the Hubbard Hamiltonian is essential in order to understand the electronic properties of the superconducting oxides and also of the important class of quasi-onedimensional conductors. ${ }^{16}$ A proper self-consistent treatment of such a model can be achieved by using variational methods. ${ }^{17,18}$ However, for practical calculations, simplified versions are preferable. The essential features on which we want to focus are present in the PeierlsHubbard Hamiltonian, ${ }^{19}$ where a bond charge-densitywave state is energetically favored in one dimension and also in two dimensions for sufficiently large electronphonon coupling. ${ }^{20}$ Dimerized states have been considered in proposed mechanisms for high- $T_{c}$ superconductivity. ${ }^{21}$

The present approximate expansion on the hopping integral is inspired by an analogous one applied by Brunet and co-workers to the periodic Anderson model. ${ }^{22}$ For the Hubbard model, similar expansions around the atomic limit have been recently developed ${ }^{23,24}$ and suggested to study a possible superconducting state with local pairing. ${ }^{23}$

In the next section, we write down the model Hamiltonian in a form appropriate to deal with a twodimensional lattice with both dimerization and anisotropy and present the diagrammatic approximation employed. In Sec. III we diagonalize the Hamiltonian on a simple two-site cluster (dimer) and obtain the corresponding one-particle Green function, from which we will construct the lattice Green function in the following section. Section V presents the calculated spectral functions, gap energy, and magnetic susceptibility. A discussion of the results is presented in the last section.

\section{MODEL HAMILTONIAN AND APPROXIMATION}

We have considered an interacting electron system on the two-dimensional lattice specified in Fig. 1. It can be regarded as a regular square lattice that has undergone a static dimerization along one of the square axes. As in the Hubbard model, ${ }^{1}$ only the on-site Coulomb repulsion $U$ is retained. However, we introduce the possibility of three different nearest-neighbor hoppings $t, t_{\|}$, and $t_{\perp}$. One assumes that this diversity may arise as the net effect 
of the electron-phonon interaction on the electron system. Without loss of generality we set $t_{\|}, t_{\perp} \leq t$. Geometrically, we call a dimer each pair of neighboring sites connected by a $t$ line. We see immediately that the positions of these dimers define another square lattice as drawn in Fig. 1 with which we will be concerned throughout this paper. It seems quite natural to look at the dimers as the lattice basic building blocks. Thus we write our model Hamiltonian in the form

$$
\mathcal{H}=\sum_{i \alpha} \mathcal{H}_{i \alpha}^{0}+\sum_{i \alpha \sigma} V_{i \alpha \sigma}
$$

where

$$
\begin{aligned}
\mathscr{H}_{i \alpha}^{0}= & U\left(n_{i \alpha \downarrow}^{a} n_{i \alpha \uparrow}^{a}+n_{i \alpha \downarrow}^{b} n_{i \alpha \uparrow}^{b}\right) \\
& -t \sum_{\sigma}\left(a_{i \alpha \sigma}^{\dagger} b_{i \alpha \sigma}+b_{i \alpha \sigma}^{\dagger} a_{i \alpha \sigma}\right)-\mu \sum_{\sigma}\left(n_{i \alpha \sigma}^{a}+n_{i \alpha \sigma}^{b}\right)
\end{aligned}
$$

is its exactly solvable unperturbed part and

$$
\begin{gathered}
V_{i \alpha \sigma}=-t_{\|}\left(a_{i \alpha \sigma}^{\dagger} b_{i+1, \alpha+1, \sigma}+b_{i \alpha \sigma}^{\dagger} a_{i-1, \alpha-1, \sigma}\right) \\
-t_{1}\left(a_{i \alpha \sigma}^{\dagger} b_{i+1, \alpha \sigma}+a_{i \alpha \sigma}^{\dagger} b_{i, \alpha+1, \sigma}\right. \\
\left.\quad+b_{i \alpha \sigma}^{\dagger} a_{i-1, \alpha \sigma}+b_{i \alpha \sigma}^{\dagger} a_{i, \alpha-1, \sigma}\right)
\end{gathered}
$$

is a one-particle "interaction" term. We have denoted by $a_{i \alpha \sigma}^{\dagger}\left(a_{i \alpha \sigma}\right)$ and $b_{i \alpha \sigma}^{\dagger}\left(b_{i \alpha \sigma}\right)$ the operators that create (annihilate) an electron with spin $\sigma$ on an $a$ or $b$ site of the $i$ th dimer and $n_{i \sigma}^{c}=c_{i \sigma}^{\dagger} c_{i \sigma}(c=a$ or $b) . U$ is the on-site Coulomb repulsion, $t$ is the intradimer hopping, $t_{\|}$and $t_{\perp}$

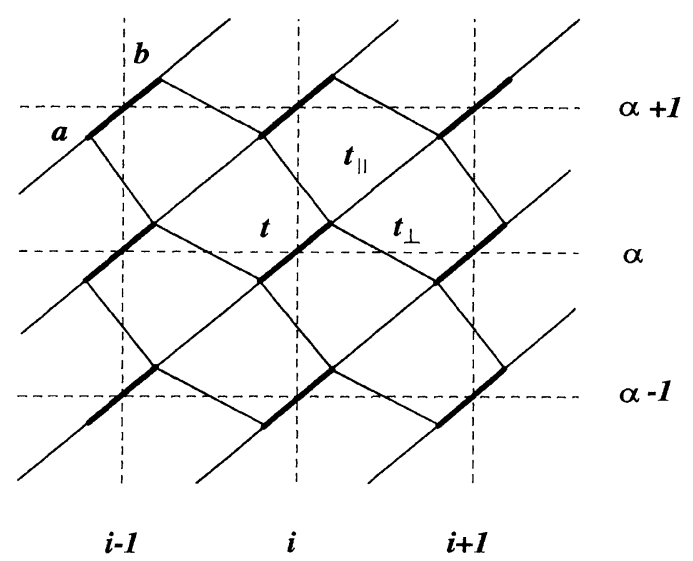

FIG. 1. The dimerized square lattice with a redefinition of the square axes.

are the hoppings between nearest-neighbor sites of different dimers as indicated in $a$ of Fig. 1 , and $\mu$ is the chemical potential.

The presence of a small, static, uniform magnetic field $h$ can be handled by adding a term

$$
\mathscr{H}_{h}=-h \sum_{\sigma} \sigma\left(n_{i \alpha \sigma}^{a}+n_{i \alpha \sigma}^{b}\right)
$$

to the unperturbed Hamiltonian $\mathscr{H}_{i \alpha}^{0}$.

The Green function that describes the propagation of an electron from a site $c$ ( $a$ or $b)$ of dimer $(i, \alpha)$ to site $d$ of dimer $(j, \beta)$ is written as

$$
G_{c d \sigma}^{i \alpha, j \beta}(\tau)=-\sum_{n=0}^{\infty}(-1)^{n} \int_{0}^{\beta} d \tau_{1} \cdots \int_{0}^{\beta} d \tau_{n 0}\left\langle T_{\tau} d_{j \beta \sigma}(\tau) \hat{V}\left(\tau_{1}\right) \cdots \hat{V}\left(\tau_{n}\right) c_{i \alpha \sigma}^{\dagger}(0)\right\rangle_{\text {dif.conn. }}
$$

where we follow the notation of Ref. 25 .

From the choice of $\mathscr{H}^{0}$ it turns out that averages of products of operators acting on sites of different dimers are decoupled. Furthermore, if one was allowed to decouple the averages at different times on the same dimer then one would be able to sum up all the remaining diagrams. This is true for $U=0$ from Wick's theorem and will be assumed here as a first approximation to the correlated case - valid at least for $U, t_{\|}, t_{\perp} \ll t$. As we are summing the perturbation terms to all orders, we expect that it will work fairly well in all parameter space-in particular, for the regular chain and square lattice.

The resulting Dyson's equation reads

$$
\begin{aligned}
& G_{c d \sigma}^{i \alpha, j \beta}(\tau)= \delta_{i j} \delta_{\alpha \beta} g_{c d}^{\sigma}(\tau) \\
&-\sum_{c^{\prime} d^{\prime} m \gamma} t_{d^{\prime} c^{\prime} \sigma}^{i, m \gamma} \int_{0}^{\beta} d \tau_{1} g_{c d^{\prime}}^{\sigma}\left(\tau-\tau_{1}\right) \\
& \times G_{c^{\prime} d \sigma}^{m \gamma, j \beta}\left(\tau_{1}\right),
\end{aligned}
$$

where $t_{d^{\prime} c^{\prime} \sigma}^{i \alpha, m \gamma}$ is equal to $t_{\|}$or $t_{\perp}$ for nearest-neighbor sites of different dimers according to Fig. 1 and zero otherwise. The bare Green functions $g_{c d}^{\sigma}(\tau)$ will be evaluated in Sec. III.

\section{THE HUBBARD DIMER}

The eigenvalues $E_{n}$ and eigenfunctions $|n\rangle$ of $\mathscr{H}_{i \alpha}^{0}+\mathscr{H}_{h}$ corresponding to the different electronic configurations are shown in Table I, where we make use of the definitions

$$
\tan \theta=(S-U / t) / 4
$$

and

$$
S=\left[(U / t)^{2}+16\right]^{1 / 2} .
$$

For half filling the ground state is $|5\rangle$, that in the limit $U / t \rightarrow \infty$ becomes $1 / \sqrt{2}\left(b_{\downarrow}^{\dagger} a_{\uparrow}^{\dagger}-b_{\uparrow}^{\dagger} a_{\downarrow}^{\dagger}\right)|0\rangle$.

The dimer's Green functions are given by the spectral representation: ${ }^{25}$

$$
\begin{aligned}
g_{c d}^{\sigma}(\omega)=\frac{1}{Q} \sum_{m, n}\left(e^{-\beta E_{m}}+e^{-\beta E_{n}}\right) \\
\times \frac{\left\langle n\left|d_{i \alpha \sigma}^{\dagger}\right| m\right\rangle\left\langle m\left|c_{i \alpha \sigma}\right| n\right\rangle}{\omega-\left(E_{n}-E_{m}\right)},
\end{aligned}
$$

where $c, d=a$ or $b, \beta=1 / k_{B} T$, and 
TABLE I. Eigenvalues and eigenfunctions of $\mathscr{H}^{0}+\mathscr{H}_{h}$.

\begin{tabular}{ll}
\hline \hline \multicolumn{1}{c}{$E_{0}$} & \multicolumn{1}{c}{$|0\rangle$} \\
\hline$E_{1}=-t-\mu-h$ & $|1\rangle=1 / \sqrt{2}\left(a_{\uparrow}^{\dagger}+b_{\uparrow}^{\dagger}\right)|0\rangle$ \\
$E_{2}=-t-\mu+h$ & $|2\rangle=1 / \sqrt{2}\left(a_{\downarrow}^{\dagger}+b_{\downarrow}^{\dagger}\right)|0\rangle$ \\
$E_{3}=t-\mu-h$ & $|3\rangle=1 / \sqrt{2}\left(a_{\uparrow}^{\dagger}-b_{\uparrow}^{\dagger}\right)|0\rangle$ \\
$E_{4}=t-\mu+h$ & $|4\rangle=1 / \sqrt{2}\left(a_{\downarrow}^{\dagger}-b_{\downarrow}^{\dagger}\right)|0\rangle$ \\
& \\
$E_{5}=U / 2-t S / 2-2 \mu$ & $|5\rangle=1 / \sqrt{2}\left\{\sin \theta\left(a_{\downarrow}^{\dagger} a_{\uparrow}^{\dagger}+b_{\downarrow}^{\dagger} b_{\uparrow}^{\dagger}\right)-\cos \theta\left(b_{\uparrow}^{\dagger} a_{\downarrow}^{\dagger}-b_{\downarrow}^{\dagger} a_{\uparrow}^{\dagger}\right)\right\}|0\rangle$ \\
$E_{6}=-2 \mu-2 h$ & $|6\rangle=b_{\uparrow}^{\dagger} a_{\uparrow}^{\dagger}|0\rangle$ \\
$E_{7}=-2 \mu$ & $|7\rangle=1 / \sqrt{2}\left(b_{\uparrow}^{\dagger} a_{\downarrow}^{\dagger}+b_{\uparrow}^{\dagger} a_{\uparrow}^{\dagger}\right)|0\rangle$ \\
$E_{8}=-2 \mu+2 h$ & $|8\rangle=b_{\downarrow}^{\dagger} a_{\downarrow}^{\dagger}|0\rangle$ \\
$E_{9}=U-2 \mu$ & $|9\rangle=1 / \sqrt{2}\left(a_{\downarrow}^{\dagger} a_{\uparrow}^{\dagger}-b_{\downarrow}^{\dagger} b_{\uparrow}^{\dagger}\right)|0\rangle$ \\
$E_{10}=U / 2+t S / 2-2 \mu$ & $|10\rangle=1 / \sqrt{2}\left\{\cos \theta\left(a_{\downarrow}^{\dagger} a_{\uparrow}^{\dagger}+b_{\downarrow}^{\dagger} d_{\uparrow}^{\dagger}\right)+\sin \theta\left(b_{\uparrow}^{\dagger} a_{\downarrow}^{\dagger}-b_{\downarrow}^{\dagger} a_{\uparrow}^{\dagger}\right)\right\}|0\rangle$ \\
& \\
$E_{11}=-t+U-3 \mu-h$ & $|11\rangle=1 / \sqrt{2} a_{\uparrow}^{\dagger} b_{\uparrow}^{\dagger}\left(a_{\downarrow}^{\dagger}+b_{\downarrow}^{\dagger}\right)|0\rangle$ \\
$E_{12}=-t+U-3 \mu+h$ & $|12\rangle=1 / \sqrt{2} b_{\downarrow}^{\dagger} a_{\downarrow}^{\dagger}\left(a_{\uparrow}^{\dagger}+b_{\uparrow}^{\dagger}\right)|0\rangle$ \\
$E_{13}=t+U-3 \mu-h$ & $|13\rangle=1 / \sqrt{2} a_{\uparrow}^{\dagger} b_{\uparrow}^{\dagger}\left(a_{\downarrow}^{\dagger}-b_{\downarrow}^{\dagger}\right)|0\rangle$ \\
$E_{14}=t+U-3 \mu+h$ & $|14\rangle=1 / \sqrt{2} b_{\downarrow}^{\dagger} a_{\downarrow}^{\dagger}\left(a_{\uparrow}^{\dagger}-b_{\uparrow}^{\dagger}\right)|0\rangle$ \\
& \\
$E_{15}=2 U-4 \mu$ & $|15\rangle=b_{\downarrow}^{\dagger} b_{\uparrow}^{\dagger} a_{\downarrow}^{\dagger} a_{\uparrow}^{\dagger}|0\rangle$ \\
\hline \hline
\end{tabular}

$$
Q=\sum_{m} e^{-\beta E_{m}}
$$

is the grand partition function.

Equation (9) yields

$$
g_{a a}^{\sigma}(\omega, h)=g_{+}^{\sigma}+g_{-}^{\sigma}
$$

and

$$
g_{a b}^{\sigma}(\omega, h)=g_{+}^{\sigma}-g_{-}^{\sigma},
$$

where we introduce for convenience

$$
g_{+}^{\sigma}(\omega, h)=\frac{1}{Q(h)} \sum_{i=1}^{4} \frac{A_{i}^{\sigma}(h)}{\omega-p_{i}^{\sigma}(h)}
$$

and

$$
g_{-}^{\sigma}(\omega, h)=\frac{1}{Q(h)} \sum_{i=5}^{8} \frac{A_{i}^{\sigma}(h)}{\omega-p_{i}^{\sigma}(h)} .
$$

TABLE II. Poles and residues of the dimer's Green functions.

\begin{tabular}{lcc}
\hline$i$ & $p_{i}^{\uparrow}(h)$ & $A_{i}^{\uparrow}(h)$ \\
\hline 1 & $t-\mu+U / 2+t S / 2-h$ & $\left(\frac{1}{4}+\frac{1}{S}\right)\left(e^{-\beta E_{13}}+e^{-\beta E_{5}}+e^{-\beta E_{10}}+e^{-\beta E_{2}}\right)$ \\
2 & $-t-\mu-h$ & $\frac{1}{2}\left(e^{-\beta E_{0}}+e^{-\beta E_{1}}+e^{-\beta E_{3}}+e^{-\beta E_{6}}\right)+\frac{1}{4}\left(e^{-\beta E_{4}}+e^{-\beta E_{7}}+e^{-\beta E_{9}}+e^{-\beta E_{11}}\right)$ \\
3 & $t-\mu+U / 2-t S / 2-h$ & $\left(\frac{1}{4}+\frac{1}{S}\right)\left(e^{-\beta E_{2}}+e^{-\beta E_{5}}+e^{\left.-\beta E_{10}+e^{-\beta E_{13}}\right)}\right.$ \\
4 & $-t-\mu+U-h$ & $\frac{1}{2}\left(e^{-\beta E_{8}}+e^{-\beta E_{12}}+e^{-\beta E_{14}}+e^{-\beta E_{15}}\right)+\frac{1}{4}\left(e^{-\beta E_{4}}+e^{-\beta E_{7}}+e^{-\beta E_{9}}+e^{-\beta E_{11}}\right)$ \\
5 & $-t-\mu+U / 2-t S / 2-h$ & $\left(\frac{1}{4}-\frac{1}{S}\right)\left(e^{-\beta E_{4}}+e^{-\beta E_{5}}+e^{-\beta E_{10}}+e^{-\beta E_{11}}\right)$ \\
6 & $t-\mu+U-h$ & $\frac{1}{2}\left(e^{-\beta E_{8}}+e^{-\beta E_{12}}+e^{-\beta E_{14}}+e^{-\beta E_{15}}\right)+\frac{1}{4}\left(e^{-\beta E_{2}}+e^{-\beta E_{7}}+e^{-\beta E_{9}}+e^{-\beta E_{13}}\right)$ \\
7 & $-t-\mu+U / 2+t S / 2-h$ & $\left.\frac{1}{4}+\frac{1}{S}\right)\left(e^{-\beta E_{11}}+e^{-\beta E_{5}}+e^{-\beta E_{10}}+e^{-\beta E_{4}}\right)$ \\
8 & $t-\mu-h$ & $\frac{1}{2}\left(e^{-\beta E_{0}}+e^{-\beta E_{1}}+e^{-\beta E_{3}}+e^{-\beta E_{6}}\right)+\frac{1}{4}\left(e^{-\beta E_{2}}+e^{-\beta E_{7}}+e^{-\beta E_{9}}+e^{-\beta E_{13}}\right)$ \\
\hline \hline
\end{tabular}


From the symmetry of the dimer, it follows that $g_{b b}^{\sigma}=g_{a a}^{\sigma}$ and $g_{b a}^{\sigma}=g_{a b}^{\sigma}$. The same will be valid for the lattice Green functions $G_{b b \sigma}^{i \alpha, j \beta}=G_{a a \sigma}^{i \alpha, j \beta}$ and $G_{b a \sigma}^{i \alpha, j \beta}=G_{a b \sigma}^{i \alpha, j \beta}$.

The poles $p_{i}$ of these Green functions correspond to the one-electron energy levels of an isolated dimer. They are shown in Table II together with their corresponding residues, defined through the matrix elements appearing in Eq. (9). For down spin we have $p_{i}^{\downarrow}(h)=p_{i}^{\uparrow}(-h)$ and $A_{i}{ }^{\downarrow}(h)=A_{i}^{\uparrow}(-h)$.

\section{THE DIMERS' LATTICE}

A Fourier transformation with respect to time is applied to Eq. (6). The equations obtained for the Green functions $G_{c d \sigma}^{i \alpha, j \beta}\left(i \omega_{v}\right)$ can be conveniently grouped in matrix form:

$$
\begin{aligned}
\underline{G}_{\sigma}^{i \alpha, j \beta}\left(i \omega_{\nu}\right)= & \underline{g}_{\sigma}\left(i \omega_{\nu}\right) \delta_{i j} \delta_{\alpha \beta} \\
& -\underline{g}_{\sigma}\left(i \omega_{v}\right) \sum_{m \gamma} \underline{T}^{i \alpha, m \gamma} \underline{G}_{\sigma}^{m \gamma, j \beta}\left(i \omega_{\nu}\right),
\end{aligned}
$$

where

$$
\begin{aligned}
& \underline{G}_{\sigma}^{i \alpha, j \beta}\left(i \omega_{v}\right)=\left[\begin{array}{ll}
G_{a a \sigma}^{i \alpha, j \beta}\left(i \omega_{v}\right) & G_{a b \sigma}^{i \alpha, j \beta}\left(i \omega_{v}\right) \\
G_{b a \sigma}^{i \alpha, j \beta}\left(i \omega_{v}\right) & G_{b b \sigma}^{i \alpha, j \beta}\left(i \omega_{v}\right)
\end{array}\right], \\
& \underline{g}_{\sigma}\left(i \omega_{v}\right)=\left[\begin{array}{ll}
g_{a a}^{\sigma}\left(i \omega_{v}\right) & g_{a b}^{\sigma}\left(i \omega_{v}\right) \\
g_{b a}^{\sigma}\left(i \omega_{v}\right) & g_{b b}^{\sigma}\left(i \omega_{v}\right)
\end{array}\right),
\end{aligned}
$$

$$
\underline{G}_{\sigma}\left(\mathbf{k}, i \omega_{v}\right)=\frac{1}{1-\Lambda\left(k_{x} a, k_{y} a\right)} \mid \begin{gathered}
g_{+}^{\sigma}+g_{-}^{\sigma} \\
g_{+}^{\sigma}-g_{-}^{\sigma}-4 g_{+}^{\sigma} g_{-}^{\sigma} \varepsilon(\mathbf{k})
\end{gathered}
$$

where

$$
\begin{aligned}
\Lambda(x, y)= & 4\left(t_{\|}^{2}+2 t_{\perp}^{2}\right) g_{+}^{\sigma} g_{-}^{\sigma}+2 t_{\perp}\left(4 t_{\|} g_{+}^{\sigma} g_{-}^{\sigma}-g_{+}^{\sigma}+g_{-}^{\sigma}\right) \\
& \times(\cos x+\cos y) \\
+ & 2\left(4 t_{1}^{2} g_{+}^{\sigma} g_{-}^{\sigma}-t_{\|} g_{+}^{\sigma}+t_{\|} g_{-}^{\sigma}\right) \cos x \cos y \\
+ & 2\left(4 t_{\perp}^{2} g_{+}^{\sigma} g_{-}^{\sigma}+t_{\|} g_{+}^{\sigma}-t_{\|} g_{-}^{\sigma}\right) \sin x \sin y
\end{aligned}
$$

The elements of this matrix are related to the usual one-particle Green function in $\mathbf{k}$ space, which can be expressed as

$$
\begin{array}{r}
\mathcal{G}_{\sigma}\left(\mathbf{k}, i \omega_{v}\right)=1 / 2\left\{G_{a a}^{\sigma}\left(\mathbf{k}, i \omega_{v}\right)+G_{b b}^{\sigma}\left(\mathbf{k}, i \omega_{v}\right)\right. \\
+e^{i \mathbf{k} \cdot \mathbf{R}^{a b}} G_{a b}^{\sigma}\left(\mathbf{k}, i \omega_{v}\right) \\
\left.+e^{-i \mathbf{k} \cdot \mathbf{R}^{a b}} G_{b a}^{\sigma}\left(\mathbf{k}, i \omega_{v}\right)\right\}
\end{array}
$$

where $\mathbf{R}^{a b}=\mathbf{R}_{i \alpha}^{a}-\mathbf{R}_{i \alpha}^{b}$.

It is straightforward to get the known results at $U=0$ for the chain and the square lattice:

$$
\begin{aligned}
& \underline{T}^{i \alpha, i+1, \alpha+1}=\underline{T}_{\|}=\left(\begin{array}{ll}
0 & t_{\|} \\
0 & 0
\end{array}\right), \\
& \underline{T}^{i \alpha, i+1, \alpha}=\underline{T}^{i \alpha, i, \alpha+1}=\underline{T}_{\perp}=\left(\begin{array}{ll}
0 & t_{\perp} \\
0 & 0
\end{array}\right), \\
& \underline{T}^{i \alpha, i-1, \alpha-1}=\widetilde{T}_{\|},
\end{aligned}
$$

and

$$
\underline{T}^{i \alpha, i-1, \alpha}=\underline{T}^{i \alpha, i, \alpha-1}=\underline{T}_{\perp},
$$

every other $\underline{T}^{i \alpha, m \gamma}$ being zero.

Equation (15) can be solved by a Fourier transformation on the dimers' lattice of $b$ of Fig. 1, which yields

$$
\underline{G}_{\sigma}^{i \alpha, j \beta}\left(i \omega_{v}\right)=\frac{1}{N} \sum_{k} \underline{G}_{\sigma}\left(\mathbf{k}, i \omega_{v}\right) e^{-i \mathbf{k} \cdot\left(\mathbf{R}_{i \alpha}-\mathbf{R}_{j \beta}\right)}
$$

with

$$
\underline{G}_{\sigma}\left(\mathbf{k}, i \omega_{v}\right)=\left[\mathbf{1}+\mathbf{g}_{\sigma}\left(i \omega_{v}\right) \underline{E}(\mathbf{k})\right]^{-1} \underline{g}_{\sigma}\left(i \omega_{v}\right),
$$

where

$$
\underline{E}(\mathbf{k})=\left[\begin{array}{cc}
0 & \varepsilon(-\mathbf{k}) \\
\varepsilon(\mathbf{k}) & 0
\end{array}\right)
$$

with

$$
\varepsilon(\mathbf{k})=t_{\|} e^{i\left(k_{x}+k_{y}\right) a}+t_{\perp}\left(e^{i k_{x} a}+e^{i k_{y} a}\right) .
$$

We obtain

$$
\left.\begin{array}{c}
g_{+}^{\sigma}-g_{-}^{\sigma}-4 g_{+}^{\sigma} g_{-}^{\sigma} \varepsilon(-\mathbf{k}) \\
g_{+}^{\sigma}+g_{-}^{\sigma}
\end{array}\right]
$$

$$
\mathcal{S}_{\sigma}^{c}\left(\mathbf{k}, i \omega_{v}\right)=\frac{1}{i \omega_{v}+2 t \cos k a_{o}}
$$

and

$$
\mathcal{G}_{\sigma}^{s}\left(\mathbf{k}, i \omega_{v}\right)=\frac{1}{i \omega_{v}+2 t\left(\cos k_{x}^{o} a_{o}+\cos k_{y}^{o} a_{o}\right)},
$$

where $a_{\rho}=a / \sqrt{2}, \quad k_{x}^{o}=\left(k_{x}-k_{y}\right) / \sqrt{2}, \quad$ and $\quad k_{y}^{o}=\left(k_{x}\right.$ $\left.+k_{y}\right) / \sqrt{2}$. tion

We are particularly interested in the local Green func-

$G_{a a \sigma}^{i \alpha, i \alpha}\left(i \omega_{v}\right)=\frac{1}{4 \pi^{2}}\left(g_{+}^{\sigma}+g_{-}^{\sigma}\right) \int_{-\pi}^{\pi} \int_{-\pi}^{\pi} d x d y \frac{1}{1-\Lambda(x, y)}$

that can be reduced to

$$
G_{a a \sigma}^{i \alpha, i \alpha}\left(i \omega_{v}\right)=\frac{2}{\pi} \frac{g_{+}^{\sigma}+g^{\sigma}}{\sqrt{R}} K(Q)
$$

where $K(z)$ is the complete elliptic integral of the first kind, 


$$
R=\left[\left(t_{1}^{2} a_{1}-a_{3}\right)^{2}+t_{1}^{2}\left(4 a_{1} a_{3}-a_{2}^{2}\right)\right]^{1 / 2},
$$

and

$$
Q^{2}=\frac{R-t_{\perp}^{2} a_{1}+a_{3}}{2 R},
$$

with

$$
\begin{aligned}
a_{1}= & -4\left(g_{+}^{\sigma}-g_{-}^{\sigma}-4 t_{\|} g_{+}^{\sigma} g_{-}^{\sigma}\right)^{2}, \\
a_{2}= & 4\left(g_{+}^{\sigma}-g_{-}^{\sigma}+4 t_{\|} g_{+}^{\sigma} g_{-}^{\sigma}\right)\left(1+2 t_{\|} g_{+}^{\sigma}\right) \\
& \times\left(1-2 t_{\|} g_{-}^{\sigma}\right), \\
a_{3}= & 4\left(t_{\perp}^{2}+t_{\|}^{2}\right)\left(g_{+}^{\sigma}-g_{-}^{\sigma}\right)^{2}+8\left(t_{\|}^{2}+2 t_{\perp}^{2}\right) g_{+}^{\sigma} g_{-}^{\sigma} \\
& -16 t_{\|}^{4}\left(g_{+}^{\sigma} g_{-}^{\sigma}\right)^{2}-1 .
\end{aligned}
$$

\section{RESULTS}

The spectral function is given by

$$
\rho_{i}^{\sigma}(\omega, h)=-\frac{1}{\pi} \lim _{\zeta \rightarrow 0} \operatorname{Im} G_{a a}^{i i \sigma}(\omega+i \xi, h) .
$$

In the absence of the perturbation, it is just a sum of $\delta$ functions located on the poles of the dimer's Green function:

$$
\rho_{i}^{\sigma}(\omega)=\frac{1}{Q} \sum_{i=1}^{8} A_{i}^{\sigma} \delta\left(\omega-p_{i}^{\sigma}\right) .
$$

As the hopping between dimers is turned on, each of these $\delta$ functions is replaced by a distribution determined from Eq. (15).

When $U=0$, our results are exact and reproduce, in particular, the nondimerized case of Ref. 26. The spectral functions obtained when dimerization is included are shown in Fig. 2, where we have chosen $t_{\|}=0.8 t$. They have been plotted only for $\omega>0$, because $\rho_{i}^{\sigma}(\omega)$ is an even function in the half-filled band case. The energy $\omega$ is measured with respect to the chemical potential $\mu$ and the energy scale is defined by $t=1$. These curves differ from those of Ref. 26 basically by the presence of a Peierls gap at the Fermi level. In one dimension $\left(t_{\perp}=0\right)$,

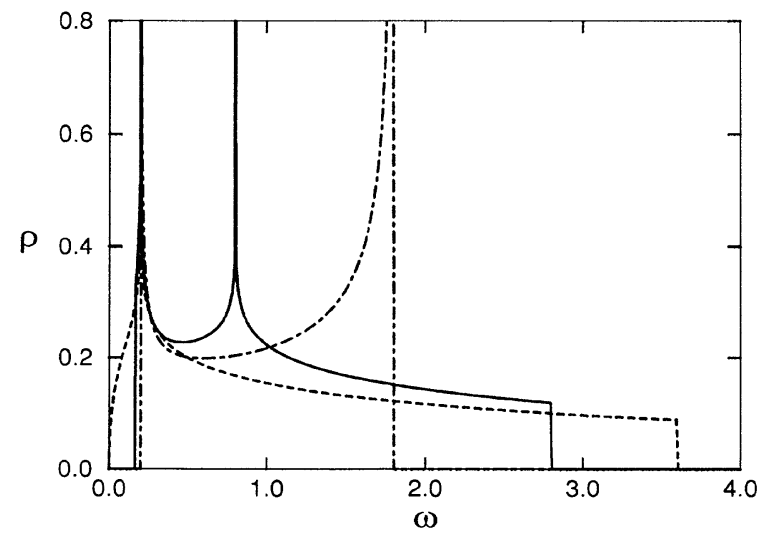

FIG. 2. Spectral functions for $U=0$ and $t_{\|}=0.8 t$, with $t_{\perp}=0$ (dot-dashed), $0.5 t$ (solid), and $0.9 t$ (dashed).

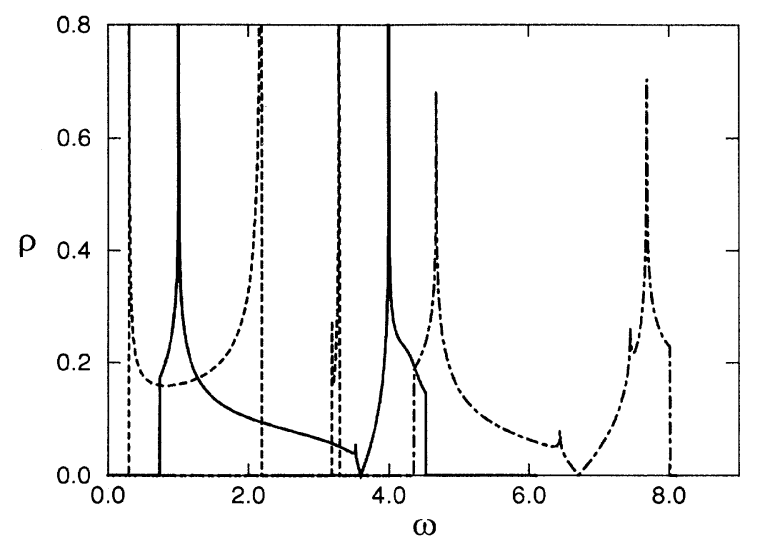

FIG. 3. Spectral functions of the linear chain for $U=2 t$ (dashed) and of the square lattice for $U=4 t$ (solid) and $U=12 t$ (dot-dashed).

there are singularities exactly at the boundaries of the zones where $\rho_{i}^{\sigma}(\omega)=0$. When $t_{\perp} \neq 0$ this is no longer true, and one can even find a vanishing gap width when $t_{\perp} \geq\left(t+t_{\|}\right) / 2$. In this case the spectral function is equal to zero at the Fermi level, but increases with infinite derivative as soon as we move away from that energy.

In Fig. 3 we plot the spectral functions of the regular chain and square lattice for the same values of $U / t$ considered in other calculations. ${ }^{10,13,7}$ Exact diagonalization of a $\sqrt{8} \times \sqrt{8}$ cluster $^{10}$ and Monte Carlo (MC) simulations on an $8 \times 8$ cluster ${ }^{13}$ yield densities of states with satellite peaks (or bumps) at the same energies where we found the satellite subbands (in the curve $U=4 t$ ). Although the second-order perturbation treatment on $U$ of Ref. 7 cannot reproduce the Hubbard gap, it also gives satellite peaks nearly at the same positions (see curves $U=2 t$ and $12 t$.

Still in the simple uncorrelated case, we obtain for an isolated dimerized chain (as in Ref. 27)

$$
\rho_{i}^{\sigma}(\omega)=\frac{1}{\pi} \frac{\omega}{\left\{\left[\omega^{2}-\left(t-t_{\|}\right)^{2}\right]\left[\left(t+t_{\|}\right)^{2}-\omega^{2}\right]\right\}^{1 / 2}}
$$

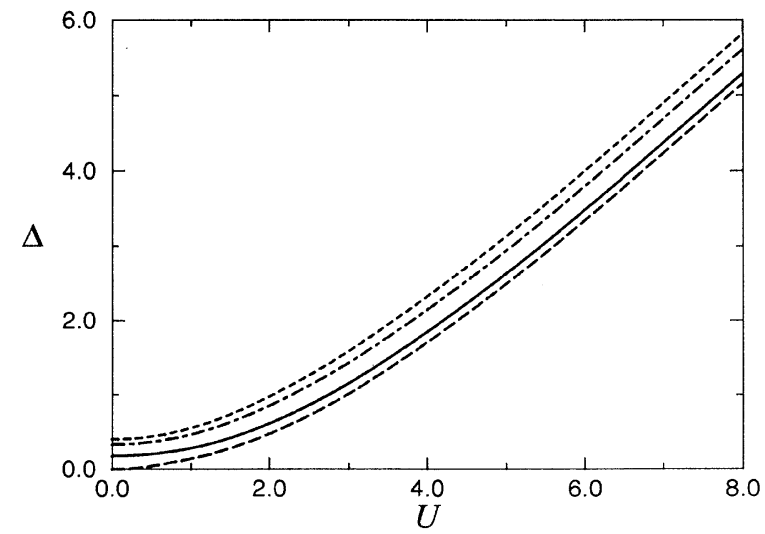

FIG. 4. Peierls-Hubbard gap as a function of $U$ for $t_{\|}=0.8 t$ with $t_{1}=0$ (dashed), $0.5 t$ (dot-dashed), $0.8 t$ (solid), and $0.9 t$ (long-dashed). 
if $\left(t-t_{\|}\right) \leq|\omega| \leq\left(t+t_{\|}\right)$, and $\rho_{\sigma}(\omega)=0$ otherwise. The one-dimensional Peierls gap is $\Delta=2\left(t-t_{\|}\right)$. For $U \geq 0$, Eq. (31) gives

$$
\Delta=-\left(2 t+t_{\|}\right)+\left[U^{2}+\left(4 t-t_{\|}\right)^{2}\right]^{1 / 2} .
$$

The two-dimensional Peierls-Hubbard gap $\Delta$ as a function of $U$ is shown in Fig. 4 for $t_{\|}=0.8 t$ and some choices of $t_{\perp}(=0,0.5 t, 0.8 t$, and $0.9 t)$. When the perpendicular hopping is turned on, we see that the gap width decreases in the whole range of $U$, the difference between the curves being practically constant for $U>2 t$. The different curvatures in the small $U$ region are associated with the abrupt disappearance of the gap for $t_{\perp} \geq\left(t+t_{\|}\right) / 2$ (when $\left.t_{\|}<t\right)$. When $t_{\|}=t$, the corresponding curves are similar, but all of them converge at the origin.

The magnetization on site $a$ (or $b$ ) of dimer $i$ is

$$
m(T, h)=\left\langle n_{i \uparrow}^{a}\right\rangle_{h}-\left\langle n_{i \downarrow}^{a}\right\rangle_{h},
$$

with

$$
\left\langle n_{i \sigma}^{a}\right\rangle_{h}=\int_{-\infty}^{+\infty} d \omega f(\omega) \rho_{i}^{\sigma}(\omega, h),
$$

where

$$
f(\omega)=\{\exp (\beta \omega)+1\}^{-1} .
$$

The paramagnetic susceptibility is

$$
\chi(T)=m^{\prime}(T, 0),
$$

with

$$
m^{\prime}(T, h) \equiv \frac{\partial}{\partial h} m(T, h) .
$$

For isolated dimers $\left(t_{\|}, t_{\perp}=0\right)$, one has

$$
\chi(T)=\frac{2 \beta\{\cosh (\beta t)+\exp (\beta U / 2)\}}{3 \cosh (\beta U / 2)+4 \cosh (\beta t)+\cosh (\beta t S / 2)} .
$$

This equation expresses the magnetic susceptibility of a two-atom cluster (calculated in Ref. 4).

The numerical integration of the spectral functions is lengthy because of their singularities, but can be per-

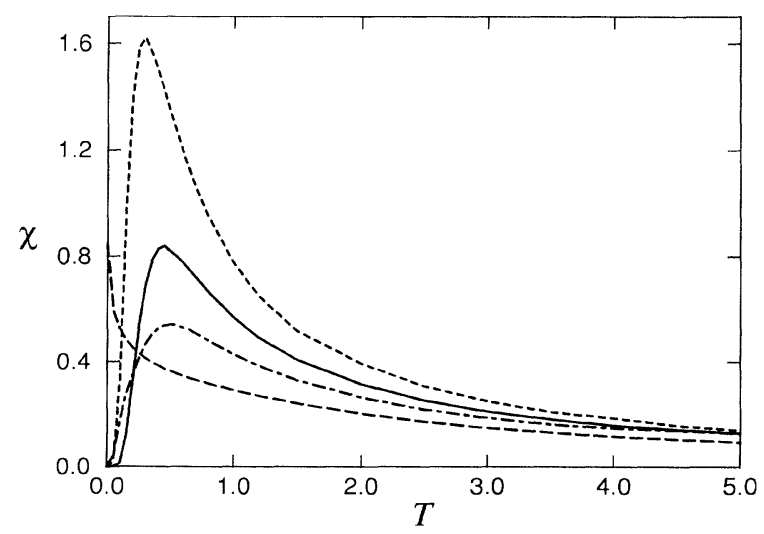

FIG. 5. Paramagnetic susceptibility of the regular square lattice for $U=0$ (long-dashed), $2 t$ (dot-dashed), $4 t$ (solid), and $8 t$ (dashed).

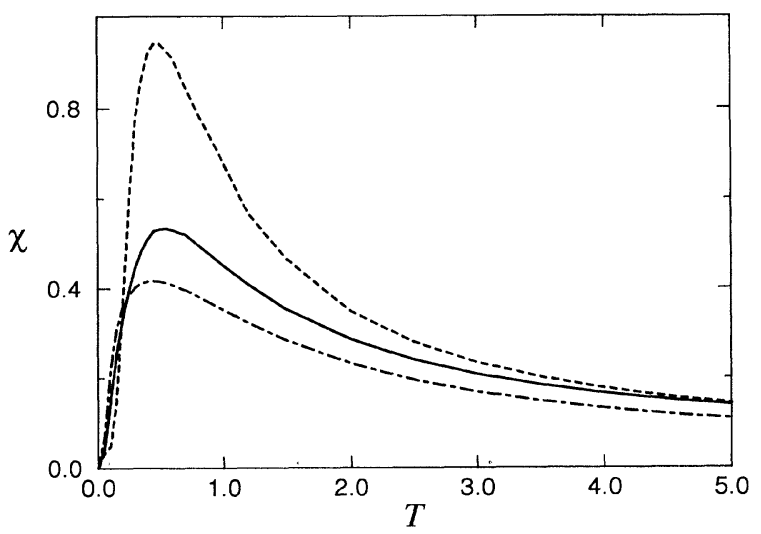

FIG. 6. Paramagnetic susceptibility of the dimerized square lattice for $t_{\|}=0.8 t, t_{\perp}=0.5 t$, and $U=0$ (dot-dashed), $t$ (solid), and $4 t$ (dashed).

formed to double precision (using extended precision). Instead of Eq. (44), it is more practical to evaluate the susceptibility from the magnetization produced by a finite, sufficiently small $h$.

Figure 5 shows the magnetic susceptibility of the square lattice (with $t_{L}=t_{\|}=t$ ) for the same values of $U / t$ considered by Hirsch in his Monte Carlo simulations on a finite cluster of $6 \times 6$ sites. $^{15}$ In Ref. 15 the magnetic susceptibility is also derived in the random-phase approximation (RPA) for $U=2 t$ and $4 t$. We see that our corresponding curves in Fig. 5 lie always between the RPA and MC ones. Thus we improve the RPA results, at least in the high-temperature region where finite-size effects are not important. For lower temperatures, we obtain a maximum structure related to the magnetic correlations present in our unperturbed Green function, though the phase transition is absent. Simulations on a larger $8 \times 8$ cluster also show a maximum but at a lower temperature. ${ }^{13}$

In Fig. 6 we plot the magnetic susceptibility of a dimerized lattice with $t_{\|}=0.8 t, t_{\perp}=\left(t+t_{\|}\right) / 2$, and some values of $U$. Comparing to the previous figure, we see that the divergency at $T=0$ for $U=0$ (exact limit) disappears as the Peierls gap develops. With increasing $U$, the maximum moves to higher temperatures. For $U=4 t$, we observe a small enhancement of $\chi$ produced by dimerization, while the maximum remains at the same position.

\section{DISCUSSION}

The satellite subbands found in our calculated spectral functions correspond to the satellite peaks reported in different approaches. ${ }^{10,13,7}$ In the present model, they originate from poles $p_{1}$ and $p_{5}$ of the dimer's Green function, which corresponds to transitions between the ground state $|5\rangle$ (of two particles) and the states with higher energy of one and three particles $(|4\rangle$ and $|13\rangle)$.

The results for the Hubbard chain $\left(t_{\|}=t, t_{1}=0\right)$ can be compared with those given by means of the Bethe ansatz. The Hubbard gap $\Delta$ was calculated by Lieb and $\mathrm{Wu}^{2}$ and rewritten in a more convenient form by Ovchinnikov. ${ }^{29}$ It behaves like 


$$
\Delta \approx\left\{\begin{array}{l}
U-4 t+\frac{8 t^{2}}{U} \ln 2 \text { for large } U, \\
\frac{8}{\pi} \sqrt{t U} \exp (-2 \pi t / U) \text { for small } U .
\end{array}\right.
$$

Here we obtain [from Eq. (40)]

$$
\Delta \approx\left\{\begin{array}{l}
U-3 t+\frac{4.5 t^{2}}{U} \text { for } U \gg 3 t, \\
\frac{U^{2}}{6 t} \text { for } U \ll 3 t .
\end{array}\right.
$$

The results agree qualitatively, though in our case the gap is somewhat greater than the exact one in the whole range of $U$, the difference going to zero as $U \rightarrow 0$. It must be noted that our result is constrained to the paramagnetic phase.

The magnetic susceptibility has been computed by Kawakami, Usuki, and Okiji ${ }^{28}$ for $U=8 t$. A direct comparison shows that the peak is too high, but its position is correctly reproduced. Such enhancement comes from the intrinsic dimerization assumed in the treatment whose effect remains even when we set $t_{\|}=t$. MC simulations for $U=8 t$ (Ref. 15) show that also in two dimensions our calculated susceptibilities present higher values. This indicates that the correlations neglected in our Dyson equation are relevant for the nondimerized lattice with large $U$.
In order to recover the antiferromagnetic order present in the ground state of the undoped, nondimerized Hubbard chain, one should introduce the average number of particles of given spin as a parameter in the unperturbed Green functions, to be determined self-consistently together with the lattice Green functions. Such a magnetic state certainly would yield a smaller Peierls-Hubbard gap (closer to the exact one) because the spectral functions for different spins would be shifted.

A better description of electronic correlations can be achieved by a higher-order approximation to the generalized Wick theorem. Attempts in this direction are in progress $^{30}$ starting from the atomic expansion where less complicated graphs are involved (see Ref. 24).

In the non-half-filled band case, the chemical potential for a given number of particles must be determined by an integral equation. Preliminary results show a transition to a metallic state upon doping with an important charge transfer between the upper and lower subbands.

\section{ACKNOWLEDGMENTS}

We are grateful to B. Coqblin, J. Perez-Conde, and M. A. C. Gusmão for helpful discussions. This work was supported by Brazilian agencies Conselho Nacional de Desenvolvimento Científico e Tecnológico $(\mathrm{CNPq})$ and Coordenação de Aperfeiçoamento de Pessoal de Nível Superior (CAPES) through Brazil-France agreement CAPES-COFECUB.
${ }^{1}$ J. Hubbard, Proc. R. Soc. London, Ser. A 276, 238 (1963).

${ }^{2}$ E. H. Lieb and F. Y. Wu, Phys. Rev. Lett. 20, 1445 (1968).

${ }^{3}$ H. Shiba and P. A. Pincus, Phys. Rev. B 5, 1966 (1977).

${ }^{4}$ H. Shiba, Prog. Theor. Phys. 48, 2171 (1972).

5J. Callaway, D. P. Chen, and R. Tang, Phys. Rev. B 35, 3705 (1987).

${ }^{6}$ P. W. Anderson, Science 235, 1196 (1987).

${ }^{7}$ H. Schweitzer and G. Czycholl, Z. Phys. B 83, 93 (1991).

${ }^{8}$ V. J. Emery, Phys. Rev. Lett. 58, 2794 (1987).

${ }^{9}$ F. C. Zhang and T. M. Rice, Phys. Rev. B 37, 3759 (1988); C.

L. Kane, P. A. Lee, and N. Read, ibid. 39, 6880 (1989).

${ }^{10}$ E. Dagotto et al., Phys. Rev. B 41, 9049 (1990).

${ }^{11}$ E. Dagotto, F. Ortolani, and D. Scalapino, Phys. Rev. B 46, 3183 (1992); E. Dagotto et al., Phys. Rev. Lett. 67, 1918 (1992); G. Chiappe et al., Phys. Rev. B 46, 3506 (1992); W. Stephan and P. Horsch, Phys. Rev. Lett. 66, 2258 (1992).

${ }^{12}$ M. Imada and Y. Hatsugai, J. Phys. Soc. Jpn. 58, 3752 (1989).

${ }^{13}$ S. R. White et al., Phys. Rev. B 40, 506 (1989).

${ }^{14}$ S. R. White et al., Phys. Rev. Lett. 63, 1523 (1989).

15J. E. Hirsch, Phys. Rev. B 31, 4403 (1985); Phys. Rev. Lett. 51, 1900 (1983).

${ }^{16}$ A. J. Berlinsky, Rep. Prog. Phys. 42, 1243 (1979); A. J. Heeger et al., Rev. Mod. Phys. 60, 781 (1988); J. T. Gammel et al.,
Phys. Rev. B 45, 6408 (1992), and references therein.

${ }^{17}$ H. Zheng, D. Feinberg, and M. Avignon, Phys. Rev. B 41, 11557 (1990).

${ }^{18}$ K. Nasu, J. Phys. Condens. Matter 4, 3793 (1992).

${ }^{19}$ J. E. Hirsch, Phys. Rev. B 35, 8726 (1987).

${ }^{20}$ F. C. Zhang and P. Prelovsek, Phys. Rev. B 37, 1569 (1988).

${ }^{21}$ M. Imada, J. Phys. Soc. Jpn. 60, 1877 (1991).

${ }^{22}$ L. G. Brunet, R. M. Ribeiro-Teixeira, and J. R. Iglesias, J. Phys. (Paris) Colloq. 49, C8-697 (1988); L. G. Brunet, M. A. C. Gusmão, and J. R. Iglesias, Phys. Rev. B 46, 4520 (1992).

${ }^{23}$ N. N. Bogolyubov and V. A. Moskalenko, Dokl. Akad. Nauk SSSR 316-321, 1107 (1991) [Sov. Phys. Dokl. 36, 160 (1991)].

${ }^{24}$ W. Metzner, Phys. Rev. B 43, 8549 (1991).

${ }^{25}$ G. D. Mahan, Many Particle Physics (Plenum, New York, 1990).

${ }^{26}$ K. Pesz and R. W. Munn, J. Phys. 19, 2499 (1986).

${ }^{27}$ W. P. Su, J. R. Schrieffer, and A. J. Heeger, Phys. Rev. B 22, 2099 (1980); Phys. Rev. Lett. 42, 1698 (1979).

${ }^{28}$ N. Kawakami, T. Usuki, and A. Okiji, Phys. Lett. A 137, 287 (1989).

${ }^{29}$ A. A. Ovchinnikov, Zh. Eksp. Teor. Fiz. 57, 2137 (1969) [Sov. Phys. JETP 30, 1160 (1970)].

${ }^{30}$ L. Craco and M. A. Gusmão (private communication). 